EPJ Web of Conferences 44, 03001 (2013)

DOI: $10.1051 /$ epjconf/20134403001

(C) Owned by the authors, published by EDP Sciences, 2013

\title{
Large deviations and heterogeneities in driven or non-driven glassy systems
}

\author{
Estelle Pitard ${ }^{1}$ \\ ${ }^{1}$ CNRS/ Université Montpellier 2, Laboratoire Charles Coulomb, 34090 Montpellier, France
}

\begin{abstract}
We give a short overview of the results for large deviations of dynamical quantities obtained for models of glassy systems. We introduce the paper with the study of kinetically constrained models (KCMs), first without external forcing. In these models, it has been shown using the thermodynamic formalism for histories, that there is a coexistence between an active and an inactive phase. Later, it has been found that adding a driving field to a KCM model leads to a singularity in the large deviation function of the current at large fields. Finally we report on recent studies on realistic glassy systems, and open directions for future research.
\end{abstract}

\section{Introduction}

Glasses are still a challenge for theorists. After decades of active research, it has become evident that structural and static differences between supercooled liquids and glasses are minute, and that dynamics are essential to understand the nature of glasses. Many studies rely on theories based on a complex energy landscape to account for both thermodynamic and dynamical features (see [1]). Here, we review on results obtained in the framework of simple models (KCMs- Kinetically Constrained Models) for which no consideration on energy is needed. These models [2], for which the focus is put on dynamical rules, allow for the systematic study of relevant order parameters for spacetime trajectories such as the activity $K(t)$ or the integrated current $Q(t)$, and of their fluctuations, more precisely their large deviation function.

In the stationary state of these models, there is a coexistence between active and inactive trajectories. These trajectories can be probed by tuning an external parameter $s$, which plays the role of a chaoticity temperature. In meanfield as well as in finite dimensional models, this translates into a singularity in the large deviation function for the activity at $s=0$. This is a first-order transition, characterized by a related discontinuity in the average activity.

The study of driven KCMs reveals the same kind of dynamical phase transitions, for the activity as well as for the integrated current; moreover it becomes more evident to relate this transition to microscopic spatial heterogeneities of the current at large fields.

Finally we will present recent results for large deviations in realistic glasses, for which the computational task remains a challenge.

\section{Glassy lattice models}

The study of large deviations for dynamical quantities is now a subject of expanding interest. It concerns the study of out-of-equilibrium models, the simplest ones being the SEP (Symmetric Exclusion Process) and ASEP (Asymmetric Exclusion Process) and can be applied to chaotic systems, dissipative systems, turbulence..

Is has been realized recently that the large deviation approach can encompass both the equilibrium thermodynamics ensemble formalism, and a thermodynamics formalism of space-time trajectories which can describe outof-equilibrium properties [3]. In this thermodynamics formalism of histories, the theory is easier to formulate and results are more tractable if the models are Markovian [4, 5, 18]. Apart from analytical results which are scarce in finite dimensions (see however $[6,7]$ ), one has to rely on different numerical methods for the computation of large deviations and cumulants of the dynamical observables. These are Trajectory Path Sampling (TPS)[15], cloning algorithm [16, 17], and density-matrix renormalization group [19-21].

We briefly present here the formalism that can be used for mean-field and finite-dimensional KCMs [11, 12] for which a first-order dynamical phase transition is found. Let us mention some other works on disordered systems, such as spin glasses [8, 9], and REM (Random Energy Model)[10] for which phase transitions between active and inactives states are also found.

KCMs are spin models on a lattice (or lattice gases), designed to mimic steric effects in amorphous materials. On each node of the lattice one puts a spin variable (or, equivalently, an occupation number) which can take two possible values. The value $s_{i}=1$, or $n_{i}=1$ defines a mobile/active state (which corresponds, in a coarse-grained view of an amorphous material, to a region of low density, with fast dynamics). If $s_{i}=-1, n_{i}=0$, one has on the con- 
trary a blocked/inactive state (region of high density, and slow dynamics). There is no interaction between spins but the dynamical evolution is dictated by specific dynamical rules. In the simplest case of the Fredrickson-Andersen (FA) model in 1 dimension: a spin can flip only if at least one of its nearest neighbours is in the mobile state. In particular, such transitions $\downarrow \uparrow \downarrow \rightleftharpoons \downarrow \downarrow \downarrow$ are forbidden. In other words, this is a diffusive system with geometric dynamical constraints. In such systems, it is known that active and inactive particles self-organize in space, leading to a slow, glassy relaxation.

How to classify time-trajectories and their activity? Following [4] one defines the activity $K(t)$ of a trajectory as the number of flips between 0 and $t$, given a history, namely a succession of configurations $C_{0} \rightarrow$ $C_{1} \rightarrow . . \rightarrow C_{t}$. For a Markovian system, the evolution of the occupation probability follows a master equation: $\frac{\partial P}{\partial t}(C, t)=\sum_{C^{\prime}} W\left(C^{\prime} \rightarrow C\right) P\left(C^{\prime}, t\right)-r(C) P(C, t)$, where $r(C)=\sum_{C^{\prime} \neq C} W\left(C \rightarrow C^{\prime}\right)$ and $W \mathrm{~s}$ are the transition rates. If one introduces $s$ (analog of a temperature), conjugated to $\mathrm{K}$, and define $\hat{P}(C, s, t)=\sum_{K} e^{-s K} P(C, K, t)$, one obtains a new evolution equation for $\hat{P}(C, s, t)$ and the generating function of K is simply $Z_{K}(s, t)=\sum_{C} \hat{P}(C, s, t)=<e^{-s K}>$. For $t \rightarrow \infty, Z_{K}(s, t) \simeq e^{t \psi_{K}(s)}$, where $\psi_{K}(s)$ is the large deviation function for the activity $K$.

The analogy with the canonical ensemble of standard thermodynamics is straightforward. In the space of configurations, at fixed $\beta, Z(\beta)=\sum_{C} e^{-\beta H} \simeq e^{-N f(\beta)}, N \rightarrow \infty$, the free energy $f(\beta)$ is (minus) the large deviation for the energy. In the space of trajectories, at fixed $s: Z_{K}(s, t)=$ $\sum_{C, K} e^{-s K} P(C, K, t) \simeq e^{-t f_{K}(s)}, t \rightarrow \infty, f_{K}(s)$ is free energy for trajectories and $\psi_{K}(s)=-f_{K}(s)$ is the large deviation function for the activity. The average activity in the s-state is given by $\frac{<K>(s, t)}{N t}=-\frac{1}{N} \psi_{K}^{\prime}(s)$, and all other cumulants can be derived as well. Two distinct phases can be studied: the active phase for $\langle K>(s, t) /(N t)>0$ and $s<0$; and the inactive phase for $\langle K\rangle(s, t) /(N t)=0$ and $s>0$. The central question is to try and understand whether there is a phase transition separating those two phases.

A simple solution can be found for the mean-field $\mathrm{FA}[11,12]$. In this model, one chooses the transition rates as: $W_{i}(0 \rightarrow 1)=k^{\prime} \frac{n}{N}, W_{i}(1 \rightarrow 0)=k \frac{n-1}{N}$, where $n=\sum_{i} n_{i}$ is the number of mobiles sites. The solution can be formulated as a variational principle for $\psi_{K}(s)$, involving a Landau-Ginzburg free energy $F_{K}(\rho, s)(\rho$ : density of mobile spins) with $F_{K}(\rho, s)=-2 \rho e^{-s}\left(\rho(1-\rho) k k^{\prime}\right)^{1 / 2}+k^{\prime} \rho(1-$ $\rho)+k \rho^{2}$, and $\frac{1}{N} \psi_{K}(s)=-\min _{\rho} F_{K}(\rho, s)$. The inspection of the minima of $F_{K}(\rho, s)$ at fixed $s$ (see Figure 1) allows to find the following phase diagram: (i) for $s>0$ : an inactive phase with $\rho_{K}(s)=0, \psi_{K}(s) / N=0$; (ii) at $s=0$ : coexistence between $\rho_{K}(0)=0$ and $\rho_{K}(0)=\rho^{*}, \psi_{K}(0)=0$, this is a first order phase transition. (iii) $s<0$ : active phase, $\rho_{K}(s)>0, \psi_{K}(s) / N>0$.

The importance of the dynamical constraints can be checked by looking at the non-constrained version of the mean-field FA model, namely with transition rates: $W_{i}(0 \rightarrow 1)=k^{\prime}, W_{i}(1 \rightarrow 0)=k$, for all $i$. Then the variational free energy reads: $F_{K}(\rho, s)=-2 e^{-s}\left(\rho(1-\rho) k k^{\prime}\right)^{1 / 2}+$
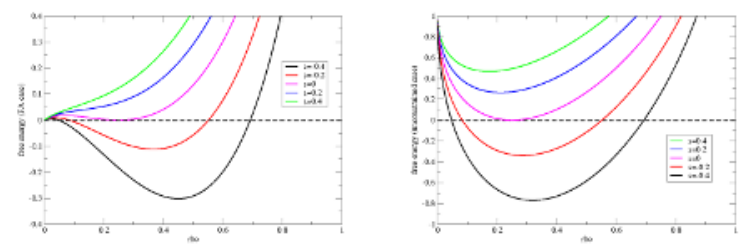

Figure 1. Left figure:mean field free energy for the FA model, two minima can be found at $s=0$. Right figure: mean field free energy without dynamical constraints, there is only one minimum for all values of $\mathrm{s}$.
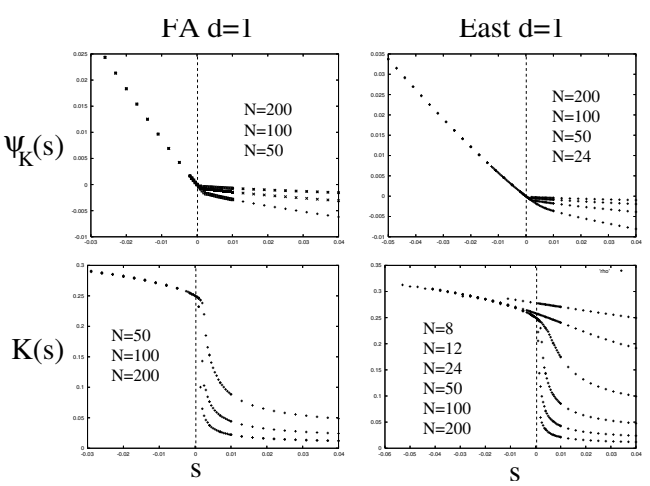

Figure 2. Large deviation function $\psi_{K}(s)$ and average activity $K(s)$ for two different KCMs: the 1d FA and the 1d East model. The finite-size study shows the convergence at large sizes towards the inactive state for $s>0$. From ref [12].

$k^{\prime}(1-\rho)+k \rho$ and no phase transition is present (see Figure $1)$.

The study of KCMs in finite dimension is a much more difficult task, and one has to use specifically-designed numerical algorithms to give a good estimation of the large deviation functions. Results were obtained in $1 \mathrm{~d}, 2 \mathrm{~d}$, and $3 \mathrm{~d}$ KCMs: in all cases a first-order transition in the activity is found at $s=0$, supported by a finite-size study, using the cloning algorithm [11, 12] (see Figure 2). It is also worth mentioning the mathematical works of $[13,14]$ which allow for a more refined finite-size scaling study of the large deviation function.

\section{Glassy lattice models with external forcing}

Driving KCMs out-of-equilibrium by an external field parameter creates a flow of particles $J$ in the system. Hence, one can study large deviations of two different dynamical quantities of interest, namely the activity $K(t)$ and the integrated current $Q(t)=\int_{0}^{t} J\left(t^{\prime}\right) d t^{\prime}$, defined as the number of moves in the direction of the field between time 0 and $t$. For a mean-field version of a driven FA model, a first-order transition is found for the entropy production, at $s=0$ in [26]. The result seems to be true also for a $2 \mathrm{~d}$ KCM studied numerically by TPS.

In [22], another model is studied, namely a $2 \mathrm{~d}$ KobAndersen model (the partcle version of FA at fixed den- 

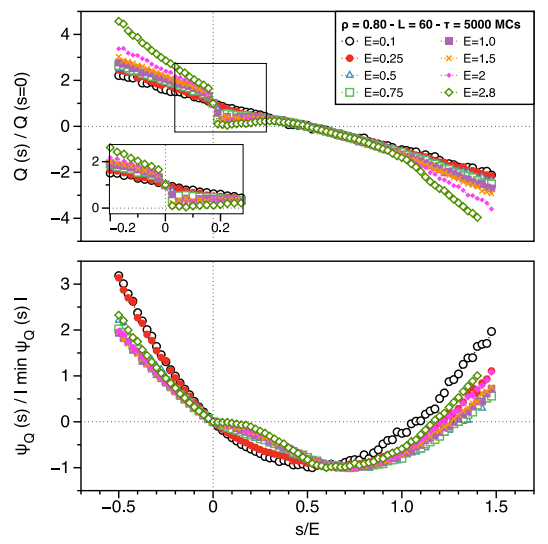

Figure 3. Large deviation function $\psi_{Q}(s)$ and average integrated current $Q(s)$ for the directed 2d KA model. For large field $E$, there is a first-order transition at $s=0$. From ref [22].

sity) on a square lettice with an external driving by a static field $E$; in other words, it is a ASEP model with dynamical constraints. The flow dependence on the field $E$ is in itself interesting: at large densities of particles, the flow is non-monotonic with $E$; it first increases with the field shear thinning- and then decreases when $E$ becomes large -shear thickening-.

The large deviation functions have been calculated numerically using the cloning algorithm, checking the validity of the results by a finite-size study. The first-order transition for the activity $K$ at $s=0$ exists for all values of the driving field, and the jump of activity between active trajectories and inactive ones increases with $E$. For the integrated current $Q$, the behaviour at small field does not apparently show a dynamic transition. However, at large fields (when the current decreases) the first-order transition is shown to occurs at $s=0$ (see Figure 3). Indeed numerical limitations start to appear for such slow systems, as computation times increase dramatically with size, time of the simulation and number of clones.

The existence of such a transition for $Q$ has the following consequence: at stationarity, there is coexistence between trajectories with a high current and trajectories with a small current. More precisely, according to the initial condition, the system can flow easily or be almost blocked. The relation between the current and microscopic features, in particular the heterogeneous, intermittent dynamics of the particles, distribution of velocities, transient shear-banding and sizes of blocking walls have been described in [23]. These allow to rationalize the average behaviour of the current as a function of the driving field. The study of the system at finite sizes [24] gives access to the dynamical correlation length, both in the shearthinning and the shear-thickening regimes. The distribution of current in a confined system is investigated as well in [24].

\section{Realistic glassy models}

It was already conjectured in $[27,28]$ that the glassy state of a supercooled liquid is characterized by the onset of the coexistence between active and inactive space-time trajectories. In these studies, this concept is illustrated by the study of the 1d FA model, where such coexistence was shown, and probability distribution functions of the activity were computed using TPS and umbrella sampling. The glassiness of the model can then be illustrated by the low activity tails in those pdfs.

A challenging question is whether the behaviour observed in non-driven or driven KCMs (see previous sections) is representative of what can be observed in real glasses. Experimental evidence is for the moment lacking. However, on the numerical side, new results have emerged, which seem promising though technically difficult. The study in [29] shows through simulations of a mixture of $N$ Lennard-Jones particles and TPS sampling that coexistence between active and inactive trajectories occurs at a critical value $s=s^{*}$ close to 0 . In this case the activity is defined as: $K(t)=\Delta t \sum_{t=0}^{t o b s} \sum_{j=1}^{N} \mid \vec{r}_{j}(t+\Delta t)-$ $\left.\vec{r}_{j}(t)\right|^{2}$, where $\Delta t$ corresponds to the time needed for a particle to move a distance of the order of a molecular diameter.

Whether $s^{*}$ is stricly zero or not is still an open question, which is difficult to confirm through numerical simulations, since they require a very precise finite-size study. In [25], the large deviation function for the activity was computed, using a finite-size study and the cloning algorithm. In this case, the activity, related to the escape rate of a particle is defined as: $K(t)=\int_{0}^{t} d t^{\prime} V_{e f f}\left(t^{\prime}\right)$ where $V_{e f f}(t)=\sum_{i}\left[\frac{\beta}{4}\left|\mathbf{F}_{i}\right|^{2}+\frac{1}{2} \nabla_{\mathbf{r}_{i}} \cdot \mathbf{F}_{i}\right]$ and $\mathbf{F}_{i}=-\sum_{j \neq i} \nabla V\left(\mathbf{r}_{i}-\right.$ $\mathbf{r}_{j}$ ) is the force acting on particle $i$ given the interaction potential $V\left(\mathbf{r}_{i}-\mathbf{r}_{j}\right)$. Up to numerical limitations, the transition seems to occur at $s^{*}$ equal to zero (see Figure 4). However, the difference of definition of the activity observable in [29] and [25] may explain the differences found in the two studies.

In [30] a "softened" FA model was studied, designed to be more realistic than the pure FA model, as it relaxes the dynamical constraints so that motion is possible, at a slow rate, even in blocked regions. In this model it can be shown that the dynamical phase transition takes place at $s^{*}$ strictly positive.

Finally $[31,32]$ have investigated in more detail particle systems in order to understand the link between structure and activity in a given phase, and see how energy landscape and dynamical properties can be related (or not) in realistic glasses. Many answers are however not clear and further work will be needed to clarify the existence of a dynamical phase transition in realistic models of glasses; this will in particular include a comparison between numerical methods in the perspective of reducing long computation times.

\section{Acknowledgements}

Numerous collaborators and collegues have to be acknowledged, through their contributions and stimulating discussions: Frédéric van Wijland, Juanpe Garrahan, Vivien Lecomte, Francesco Turci, Mauro Sellitto, Kristina van Duijvanduijk, David Chandler, Chris Fullerton, Rob 


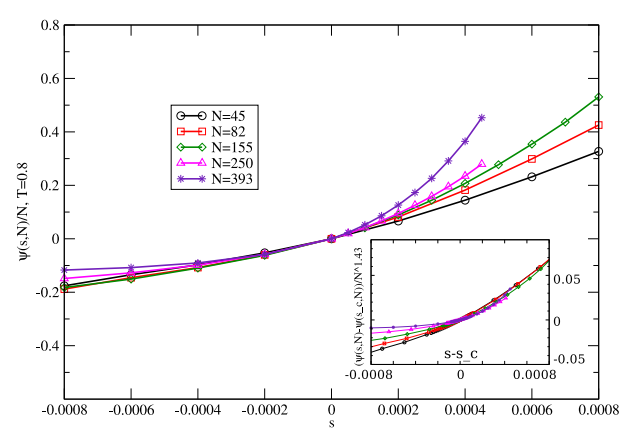

Figure 4. Large deviation function for the activity in a $3 \mathrm{~d}$ mixture of Lennard-Jones particles, from ref [25]. The finite size study hints at a transition at $s=0$.

Jack, Jorge Kurchan, Christian Maes, Kirone Mallick, Carlo Vanderzande and others.

\section{References}

[1] P.G. Debenedetti, F.H. Stillinger, Nature 410 (2001) 259.

[2] F. Ritort, P. Sollich, Adv. Phys 52, (2003) 219.

[3] H. Touchette, Phys. Rep. 478, 1 (2009).

[4] V. Lecomte, C. Appert-Rolland, F. van Wijland, Phys. Rev. Lett. 95010601 (2005).

[5] V. Lecomte, C. Appert-Rolland, F. van Wijland, J. Stat. Phys. 127 51-106 (2007).

[6] C. Appert-Rolland, B. Derrida, V. Lecomte, F. Van Wijland, Phys.Rev.E, 78 (2008) 021122.

[7] V. Lecomte, Juan P. Garrahan, F. Van Wijland, J. Phys. A, 45175001 (2012).

[8] K. van Duijvenduijk, R.L. Jack, F. van Wijland, Phys. Rev. E 81 (2010) 011110.

[9] R.L. Jack, J.P. Garrahan Phys. Rev. E 81 (2010) 011111.

[10] K. van Duijvenduijk, G. Schehr, F. van Wijland, Phys. Rev. E 78 (2008) 011120.

[11] J.P. Garrahan, R.L. Jack, V. Lecomte, E. Pitard, K. van Duijvendijk, F. van Wijland, Phys. Rev. Lett. 98, (2007) 195702.
[12] J.P. Garrahan, R.L. Jack, V. Lecomte, E. Pitard, K. van Duijvendijk, F. van Wijland, J. Phys. A 42, (2009) 075007.

[13] T. Bodineau, C. Toninelli, Com. Math.Phys. 311 357(2012).

[14] T. Bodineau, V. Lecomte, C. Toninelli, J. of Stat. Phys, 147, 1 (2012).

[15] C. Dellago, P.G. Bolhuis, P.L. Geissler, Adv. Chem. Phys. 1231 (2002).

[16] C. Giardinà, J. Kurchan, L. Peliti, Phys. Rev. Lett. 96, (2006) 120603.

[17] C. Giardina, J. Kurchan, V. Lecomte, and J. Tailleur, Journal of Statistical Physics, 145 (2011) 787.

[18] R.L. Jack, P. Sollich, Prog. Theor. Phys. Supp. 184,(2010) 200.

[19] M. Gorissen, J. Hooyberghs, and C. Vanderzande, Phys. Rev. E, 79 (2009) 0201019.

[20] M. Gorissen, C. Vanderzande, J. Phys. A: Math. Theor. 44 (2011) 115005.

[21] M. Gorissen, A. Lazarescu, K. Mallick, C. Vanderzande, Phys. Rrv. Lett. 109 (2012) 170601.

[22] F. Turci, E. Pitard, EPL 94, (2011) 10003.

[23] F. Turci, E. Pitard, M. Sellitto, Phys. Rev. E 86, (2012) 031112.

[24] F. Turci, E. Pitard, Fluc. Noise Lett. 11, (2012) 1242007.

[25] E. Pitard, V. Lecomte, F. van Wijland, EPL 96 (2011) 56002.

[26] T. Speck, J.P Garrahan, Eur. Phys. J. B 79 (2011) 1.

[27] M. Merolle, J.P. Garrahan, D. Chandler, Proc. Natl. Acad. Sci. USA 102 (2005) 10837.

[28] R.L. Jack, J. P. Garrahan, D. Chandler, J. Chem. Phys. 125 (2006) 184509.

[29] L.O. Hedges, R.L. Jack, J.P. Garrahan, D. Chandler, Science, 323 (2009) 1309.

[30] Y.S. Elmatad, R.L. Jack, J.P. Garrahan, D. Chandler, Proc. Natl. Acad. Sci. USA 107, (2012) 12793.

[31] T. Speck, D. Chandler, J. Chem. Phys, 136 (2012) 184509.

[32] Robert L. Jack, Lester O. Hedges, Juan P. Garrahan, David Chandler. Phys. Rev. Lett., 107 (2011) 275702. 